\title{
Modélisation mathématique de l'évolution, à long terme, des teneurs en nitrates dans la nappe aquifère des craies du Crétacé de Hesbaye (Belgique) \\ Groundwater modelling of the long term evolution of the nitrate content in the Cretaceous chalky aquifer of Hesbaye (Belgium)
}

\author{
V. Hallet
}

Volume 17, numéro 1, 2004

URI : https://id.erudit.org/iderudit/705520ar

DOI : https://doi.org/10.7202/705520ar

\section{Aller au sommaire du numéro}

\section{Éditeur(s)}

Université du Québec - INRS-Eau, Terre et Environnement (INRS-ETE)

\section{ISSN}

0992-7158 (imprimé)

1718-8598 (numérique)

\section{Découvrir la revue}

Citer cet article

Hallet, V. (2004). Modélisation mathématique de l'évolution, à long terme, des teneurs en nitrates dans la nappe aquifère des craies du Crétacé de Hesbaye (Belgique). Revue des sciences de l'eau / Journal of Water Science, 17(1), 3-22. https://doi.org/10.7202/705520ar

\section{Résumé de l'article}

La nappe aquifère de Hesbaye, logée dans les craies du Crétacé, est sollicitée à raison de trente millions de mètres cubes par an. Bien que naturellement protégée par une épaisseur de 5 à 20 mètres de limons, de nombreux indices montrent une dégradation de la qualité des eaux souterraines, notamment par les nitrates. Les concentrations en nitrates atteignent 15 à $25 \mathrm{mg} . \mathrm{l}^{-1}$ dans la partie semi-captive de la nappe et sont systématiquement supérieures à 35 mg. $l^{-1}$ dans la partie libre. Malgré de fortes fluctuations temporelles, les teneurs augmentent en moyenne de $0,35 \mathrm{mg} . \mathrm{l}^{-1}$ à $0,7 \mathrm{mg} . \mathrm{l}^{-1}$ par an selon la situation semi-captive ou libre de la nappe.

La détermination des paramètres hydrodynamiques et de transport de la craie par plus de 35 traçages répartis sur 11 sites, a permis de réaliser un modèle local $\left(10 \mathrm{~km}^{2}\right)$ de transport simulant la propagation des nitrates dans la nappe. Le modèle a montré que cette dernière est, malgré une certaine homogénéisation, très sensible aux apports de surface engendrant une très forte variation spatiale des concentrations. La nappe réagit de manière très différente selon que les apports de surface sont d'origine ponctuelle ou diffuse. Pour les pollutions ponctuelles, les concentrations fluctuent rapidement avec des valeurs maximales et minimales observées respectivement en périodes de basses eaux et de hautes eaux. Cette situation est liée à un phénomène de dilution de la pollution par les eaux en provenance de l'amont. En cas de suppression d'une pollution ponctuelle, la qualité de la nappe s'améliore rapidement (délai de 1 à 2 ans). Pour les pollutions diffuses, les concentrations minimales s'observent en période de rabattement de la nappe : le front de nitrates migre plus lentement (environ 1 à $2 \mathrm{~m}$ par an) que les vitesses de rabattement de la nappe (jusqu'à $5 \mathrm{~m}$ par an) et les intrants restent nuls durant des périodes pouvant aller jusqu'à 3 ans.

Différentes simulations mathématiques ont montré que si la quantité d'intrants d'origine diffuse diminue de manière permanente, la nappe mettra une vingtaine d'années pour se rééquilibrer. Ces constatations sont primordiales dans le cadre de la mise en œuvre de mesures de protection puisque, si les résultats de la suppression des pollutions ponctuelles sont rapidement mais localement observés, ceux liés à la diminution des pollutions d'origine diffuse sont observés dans des délais nettement plus longs (une à deux décennies). Ces résultats montrent clairement que toute gestion qualitative des aquifères doit être basée sur des actions à long terme.
Ce document est protégé par la loi sur le droit d'auteur. L’utilisation des services d'Érudit (y compris la reproduction) est assujettie à sa politique d'utilisation que vous pouvez consulter en ligne.

https://apropos.erudit.org/fr/usagers/politique-dutilisation/ 


\title{
Modélisation mathématique de l'évolution, à long terme, des teneurs en nitrates dans la nappe aquifère des craies du Crétacé de Hesbaye (Belgique)
}

\author{
Groundwater modelling of the long term evolution \\ of the nitrate content in the Cretaceous chalky aquifer \\ of Hesbaye (Belgium)
}

\section{HALLET}

Reçu le 21 mars 2002, accepté le 1r septembre 2003*.

\section{SUMMARY}

The Hesbaye area is located in the northeastern part of Belgium. The aquifer formations consist of chalk deposits. Groundwater provides about $80,000 \mathrm{~m}^{3} \mathrm{~d}^{-1}$. Despite 5 to 20 meters of superficial loess deposits, the groundwater quality is threatened by increasing nitrate concentrations of $0.35 \mathrm{mg} \cdot \mathrm{L}^{-1}$ per year in the semi-confined part of the aquifer to $0.7 \mathrm{mg} \cdot \mathrm{L}^{-1}$ in the unconfined aquifer. Presently, nitrate concentrations are between 15 and $25 \mathrm{mg} \cdot \mathrm{L}^{-1}$ in the semi-confined part of the aquifer but are more than $35 \mathrm{mg} \cdot \mathrm{L}^{-1}$ (reaching locally $150 \mathrm{mg} \cdot \mathrm{L}^{-1}$ ) in the unconfined part that covers $95 \%$ of the area. Nitrate concentrations have such a high spatial variation that various statistical treatments (such as kriging used to draw iso-concentration maps) have failed. This failure is due to the fact that the concentrations are highly influenced by surface land use (grass land, culture land, villages, point source pollutants, etc.). In addition, nitrate content in the aquifer varies vertically with decreasing values at depth (gradient of $0.7 \mathrm{mg} \cdot \mathrm{L}^{-1} \cdot \mathrm{m}^{-1}$ ).

Aquifer parameters were determined by 38 pumping and tracer tests conducted in radial convergent or cylindrical flow at 11 sites. Results showed that hydraulic conductivity values ranged from $1 \cdot 10^{-6} \mathrm{~m} \cdot \mathrm{s}^{-1}$ to $4 \cdot 10^{-2} \mathrm{~m} \cdot \mathrm{s}^{-1}$ and effective porosities from $0.5 \%$ to $7 \%$, showing that the aquifer was heterogeneous. Dispersivity values were affected by scale effects and varied according to chalk weathering or fracture zones. They ranged from less than $5 \mathrm{~m}$ in fractures to more than $60 \mathrm{~m}$ in weathered chalk (as in the upper part of the aquifer) and in the chalk matrix. In the chalk, transport processes were influenced by the immobile water effect due to diffusive transfer from the moving to the non-moving fluid. Non-effective porosity filled by non-moving

Département de géologie, Facultés Universitaires Notre-Dame de la Paix, rue de Bruxelles, $n^{\circ}$ 61, 5000 Namur, Belgique. 
fluid was estimated between 8 to $42 \%$. The transfer constant ranged from $0.98 \cdot 10^{-7} \mathrm{~s}^{-1}$ to $10 \cdot 10^{-7} \mathrm{~s}^{-1}$.

The determination of the transport parameters allowed simulation of nitrate transport at a regional scale. The SUFT3D (Saturated and Unsaturated Flow and Transport Model), developed by the Hydrogeology Section of the Georesources, Geotechnologies and Building Materials Department of Liege University was used. The modelled groundwater zone was defined as a $2.0 \times 4.5 \mathrm{~km}$ rectangle of $10 \mathrm{~km}^{2}$. The aquifer was subdivided into 6 layers of 3350 cells $(50 \times 50 \mathrm{~m}$ wide and 3 to $15 \mathrm{~m}$ thick). Boundary flow conditions were defined as a prescribed head (Dirichlet conditions) to the north and the south of the area modelled. As the model simulations run for a time period of 30 years, the northern Dirichlet conditions had to be adapted to the regional and seasonal water table fluctuations that were observed during this period. At the south boundary, as the aquifer is drained by the river Geer, the water table is fixed at the river bed altitude. The eastern and western boundaries were, according to the regional piezometry, assumed to be impermeable. For the transport boundary conditions, prescribed flux (Cauchy conditions) was used for the aquifer top. Elsewhere Neumann conditions were used.

Simulations were run for the period from 1963 to 1992. Nitrate inputs were averaged yearly and estimated according to actual input conditions. These conditions were calculated by simulation of nitrate flows through the nonsaturated part of the aquifer using the EPIC-Model and taking into account the amount of nitrate fertilisers used by farmers (given by the Belgian government Statistical Institute). Initial conditions were calculated according to the 1963 nitrate inputs.

Simulations demonstrated that it is important to distinguish the origin of the pollution as either point or non-point (diffuse) sources. For point source pollutants (such as contaminated infiltration basins), aquifer nitrate concentrations increased during low water level periods due to weaker dilution linked with a poor regional water gradient. During high groundwater levels, dilution is more important and the nitrate concentration decreases. If a point source pollutant is suppressed, aquifer quality is improved within one to two years. This demonstrates the importance of protective actions that could be applied within the framework of the protection zones around collecting galleries and pumping fields.

For diffuse contamination the mean input over the area $(10 \mathrm{~m}$ depth below cropped areas) increased from $1.32 \cdot 10^{-7} \mathrm{mg} \cdot \mathrm{m}^{-2} \cdot \mathrm{s}^{-1}$ in 1963 to $5.14 \cdot 10^{-7} \mathrm{mg} \cdot \mathrm{m}^{-2} \cdot \mathrm{s}^{-1}$ (i.e., a factor of four). According to these values, concentrations ranged from $11 \mathrm{mg} \cdot \mathrm{L}^{-1}$ to $22 \mathrm{mg} \cdot \mathrm{L}^{-1}$ (i.e., increasing by $0.5 \mathrm{mg} \cdot \mathrm{L}^{-1}$ per year) between 1963 and 1992. Predictive simulations, using 1992 input, show that it will take more or less 30 years for the aquifer to be in equilibrium with the 1992 input. At that time the mean concentration value will be around $30 \mathrm{mg} \cdot \mathrm{L}^{-1}$.

The main results of the simulations clearly show that if actions are taken to decrease nitrate inputs, even if the aquifer nitrate contents rapidly react to the new input, nitrate levels will decrease slowly and take about 30 years to be in equilibrium with the new inputs. This Iong delay is due to the immobile water effect that is characteristic of the chalk aquifer. Thus it is important to inform environmentalists who work on action programs (such as the water directive imposed by the European Community in the vulnerable zones) that the effects of their actions must be based on 10 to 20 year scenarios. To this estimation, based on the reaction time of the aquifer to a new input, one must also add the time transfer of the pollutant through the unsaturated part of the aquifer.

Key words: Hebaye aquifer, chalk, nitrate contamination, groundwater modelling, immobile water effect. 


\section{RÉSUMÉ}

La nappe aquifère de Hesbaye, logée dans les craies du Crétacé, est sollicitée à raison de trente millions de mètres cubes par an. Bien que naturellement protégée par une épaisseur de 5 à 20 mètres de limons, de nombreux indices montrent une dégradation de la qualité des eaux souterraines, notamment par les nitrates. Les concentrations en nitrates atteignent 15 à 25 mg..$^{-1}$ dans la partie semi-captive de la nappe et sont systématiquement supérieures à $35 \mathrm{mg.l}^{-1}$ dans la partie libre. Malgré de fortes fluctuations temporelles, les

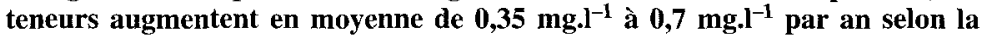
situation semi-captive ou libre de la nappe.

La détermination des paramètres hydrodynamiques et de transport de la craie par plus de 35 traçages répartis sur 11 sites, a permis de réaliser un modèle local $\left(10 \mathrm{~km}^{2}\right)$ de transport simulant la propagation des nitrates dans la nappe. Le modèle a montré que cette dernière est, malgré une certaine homogénéisation, très sensible aux apports de surface engendrant une très forte variation spatiale des concentrations. La nappe réagit de manière très différente selon que les apports de surface sont d'origine ponctuelle ou diffuse. Pour les pollutions ponctuelles, les concentrations fluctuent rapidement avec des valeurs maximales et minimales observées respectivement en périodes de basses eaux et de hautes eaux. Cette situation est liée à un phénomène de dilution de la pollution par les eaux en provenance de l'amont. En cas de suppression d'une pollution ponctuelle, la qualité de la nappe s'améliore rapidement (délai de 1 à 2 ans). Pour les pollutions diffuses, les concentrations minimales s'observent en période de rabattement de la nappe : le front de nitrates migre plus lentement (environ 1 à $2 \mathrm{~m}$ par an) que les vitesses de rabattement de la nappe (jusqu'à $5 \mathrm{~m}$ par an) et les intrants restent nuls durant des périodes pouvant aller jusqu'à 3 ans.

Différentes simulations mathématiques ont montré que si la quantité d'intrants d'origine diffuse diminue de manière permanente, la nappe mettra une vingtaine d'années pour se rééquilibrer.

Ces constatations sont primordiales dans le cadre de la mise en auvre de mesures de protection puisque, si les résultats de la suppression des pollutions ponctuelles sont rapidement mais localement observés, ceux liés à la diminution des pollutions d'origine diffuse sont observés dans des délais nettement plus longs (jusqu’à trois décennies). Cette situation est liée aux phénomènes de double porosité et d'effet d'eau immobile caractérisque du milieu crayeux.

Ces résultats montrent clairement que toute gestion qualitative des aquifères doit être basée sur des actions à long terme.

Mots clés : nappe aquifère de Hesbaye, craie, contamination par les nitrates, modélisation, effet d'eau immobile.

\section{1 - INTRODUCTION}

Le plateau de Hesbaye est situé au nord-ouest de Liège (Belgique). II est délimité par les vallées de la Mehaigne à l'ouest, de la Meuse au sud et à l'est et du Geer au nord (figure 1). Sa superficie est de $350 \mathrm{~km}^{2}$. L'aquifère est contenu dans les craies du Crétacé (DASSARGUES et MONJOIE, 1993). Annuellement, trente-deux millions de mètres cubes sont prélevés dans cette nappe à partir de $56 \mathrm{~km}$ de galeries captantes et d'une douzaine de puits. 


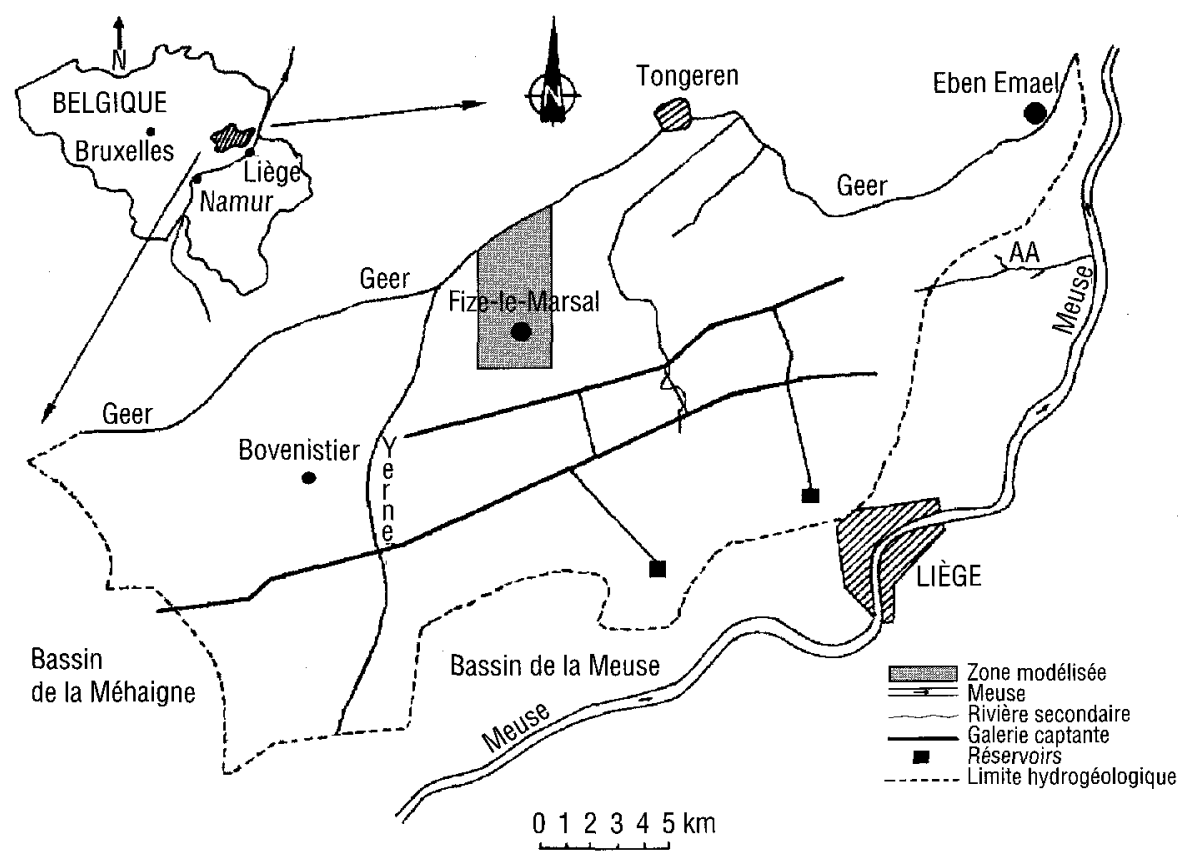

Figure 1 Carte de localisation.

Location map.

La région est une zone de " grandes cultures intensives ". Suite à l'augmentation constante des teneurs en nitrates dans la nappe, le Ministère de la Région wallonne a classé la nappe de Hesbaye en "zone vulnérable " telle que définie par la Directive européenne concernant la protection des eaux contre la pollution par les nitrates à partir de sources agricoles (91-676/CEE).

La directive européenne impose la mise en place, par un « programme d'actions ", de mesures de protection au droit de ces zones. L'objectif de ces mesures est de diminuer les intrants et, in fine, les concentrations en nitrates dans la nappe. Afin de garantir la pérennité et donc l'efficacité de telles actions, il convenait d'estimer le temps nécessaire pour que les effets des mesures mises en place sur la qualité des eaux souterraines puissent être observés.

\section{2 - CONTEXTE HYDROGÉOLOGIQUE}

Les formations géologiques sont constituées (figure 2), de bas en haut :

- la smectite de Herve du Campanien inférieur : marne riche en argiles gonflantes constituant la base imperméable de l'aquifère ;

- la craje du Campanien : craie blanche compacte, épaisse de 20 à $40 \mathrm{~m}$, généralement peu altérée sauf au droit des axes d'écoulement préférentiel localisés dans les vallées sèches (HALLET et al., 1997) ; 


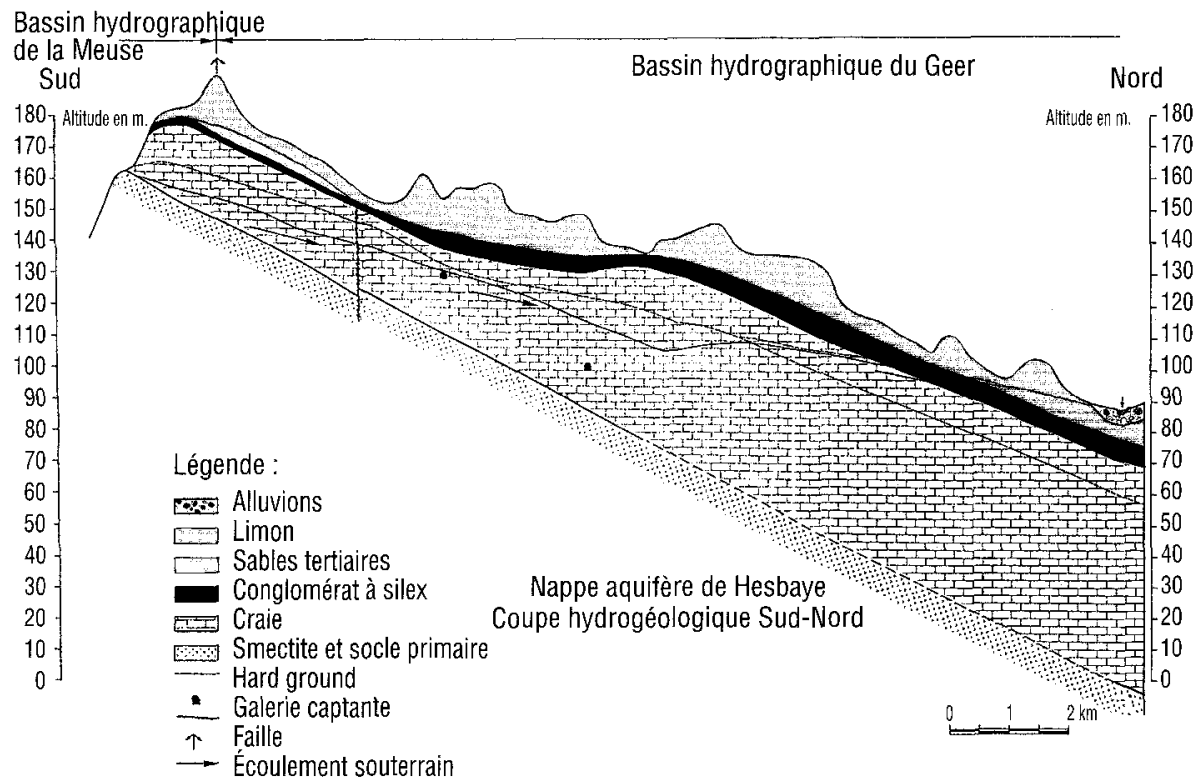

Figure 2 Coupe géologique sud-nord.

South-north geological profile.

- le hard-ground : banc de craie indurée, épais de 1 à $2 \mathrm{~m}$, peu perméable mais discontinu marquant une discordance entre le Campanien et le Maastrichtien ;

- la craie du Maastrichtien : craie grise, plus altérée et riche en silex, épaisse de 10 à $15 \mathrm{~m}$;

- le conglomérat à silex : constitué de résidus de dissolution de la craie, épais de 2 à $10 \mathrm{~m}$.

Ces formations sont recouvertes localement de dépôts sablo-argileux du Sélandien et du Priabonien, de lambeaux de sables et d'argiles oligocènes et de limons quaternaires. Ces derniers, d'origine éolienne, sont épais de 5 à $20 \mathrm{~m}$.

En raison de l'allure géométrique du réservoir (bancs réguliers d'orientation est - ouest à léger pendage vers le nord) et de la disposition des limites du bassin, la nappe présente un écoulement cylindrique du sud vers le nord en direction du Geer (principal exutoire de la nappe). Le gradient est de l'ordre de $1 \%$ au sud des galeries de captage, de $0,5 \%$ au nord et de $0,3 \%$ à proximité du Geer. La nappe est généralement libre sauf dans la plaine alluviale du Geer (figure 2) où elle est semi-captive sous les limons et les alluvions et à l'ouest où elle est localement captive sous les dépôts argileux du Sélandien.

L'étude des niveaux piézométriques saisonniers montre que le niveau de la nappe fluctue de manière pluriannuelle (cycle de plus ou moins 10 ans) avec une amplitude pouvant atteindre $17 \mathrm{~m}$ dans sa partie centrale mais s'atténuant vers le nord où le Geer impose une piézométrie constante égale au niveau d'eau dans la rivière. L'étude par corrélogrammes croisés (MANGIN, 1984) des 
niveaux piézométriques avec les infiltrations efficaces montre un retard à l'infiltration variant de quelques semaines (partie sud de la nappe) à 19 mois (partie centrale de l'aquifère) en fonction de l'épaisseur et la perméabilité des terrains de couverture (limon, sable, conglomérat).

\section{3 - ASPECT QUALITATIF}

L'inventaire des données hydrochimiques montre que, dans la partie libre de la nappe, les concentrations en nitrates sont supérieures à $35 \mathrm{mg.l}^{-1}$ (atteignant localement plus de $150 \mathrm{mg}^{-l^{-1}}$ ) ; dans la partie semi-captive, elles varient

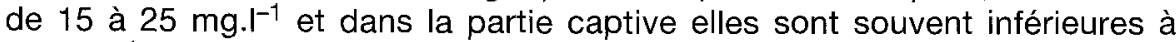
$10 \mathrm{mg.l}^{-1}$. Depuis 1975 , les teneurs moyennes annuelles en nitrates augmentent de l'ordre de $0,7 \mathrm{mg} . \mathrm{I}^{-1}$ par an dans la partie libre de la nappe et de $0,35 \mathrm{mg} . \mathrm{I}^{-1}$ par an dans la partie semi-captive (figure 3 ).

Outre cette tendance à l'augmentation, des prélèvements réguliers ont démontré que les teneurs en nitrates sont fortement dépendantes des fluctuations piézométriques de la nappe avec des valeurs minimales en période de basses eaux et des valeurs maximales en période de hautes eaux. L'amplitude

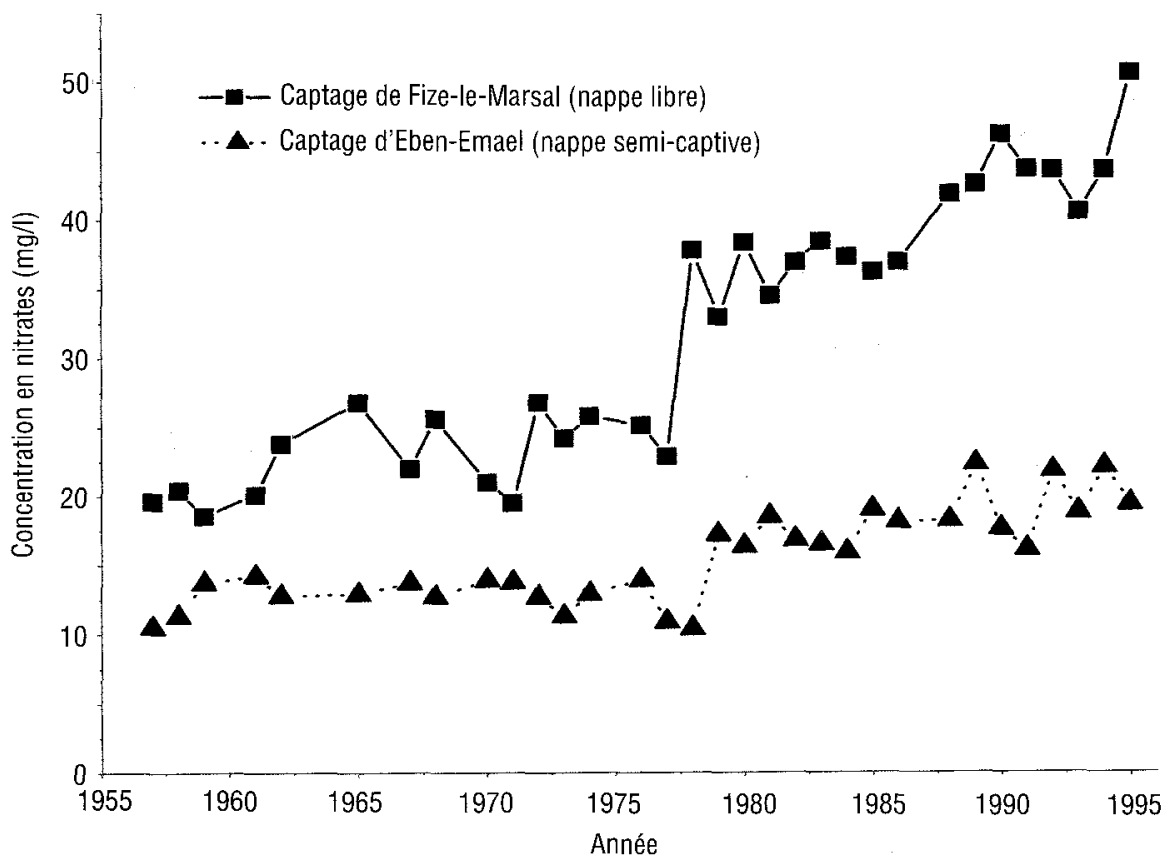

Figure 3 Évolution des concentrations en nitrates dans la nappe de Hesbaye.

Evolution of nitrate content in the Hesbaye aquifer. 
des fluctuations piézométriques peut atteindre plus de $10 \mathrm{~m}$ (notamment durant la période de sécheresse 1992 - 1993). Sur certains sites, les teneurs en nitrates peuvent varier de $37,8 \mathrm{mg} . \mathrm{I}^{-1}$ en période de basses eaux à $124 \mathrm{mg} . \mathrm{I}^{-1}$ en période de hautes eaux. Ce phénomène est lié au décrochement du niveau piézométrique de la nappe, dont la vitesse de rabattement de $0,6 \mathrm{~m}$ par mois (HALLET, 1999a) est plus rapide que le front de nitrates descendant dont les vitesses de migration sont estimées entre 0,10 et $0,20 \mathrm{~m}$ par mois (DAUTREBANDE et al., 1999). L'effet d'eau immobile (BEAR et BACHMAT, 1990) caractéristique de la double porosité des craies, accentue également les variations saisonnières de concentration. L'effet d'eau immobile, particulièrement important dans les craies, a notamment été mis en évidence et quantifié par de très nombreux traçages caractérisés par de lentes décroissances des concentrations de la courbe de restitution.

Des prélèvements effectués au droit d'un même site ont également mis en évidence une diminution des concentrations en profondeur selon un gradient

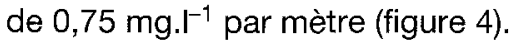

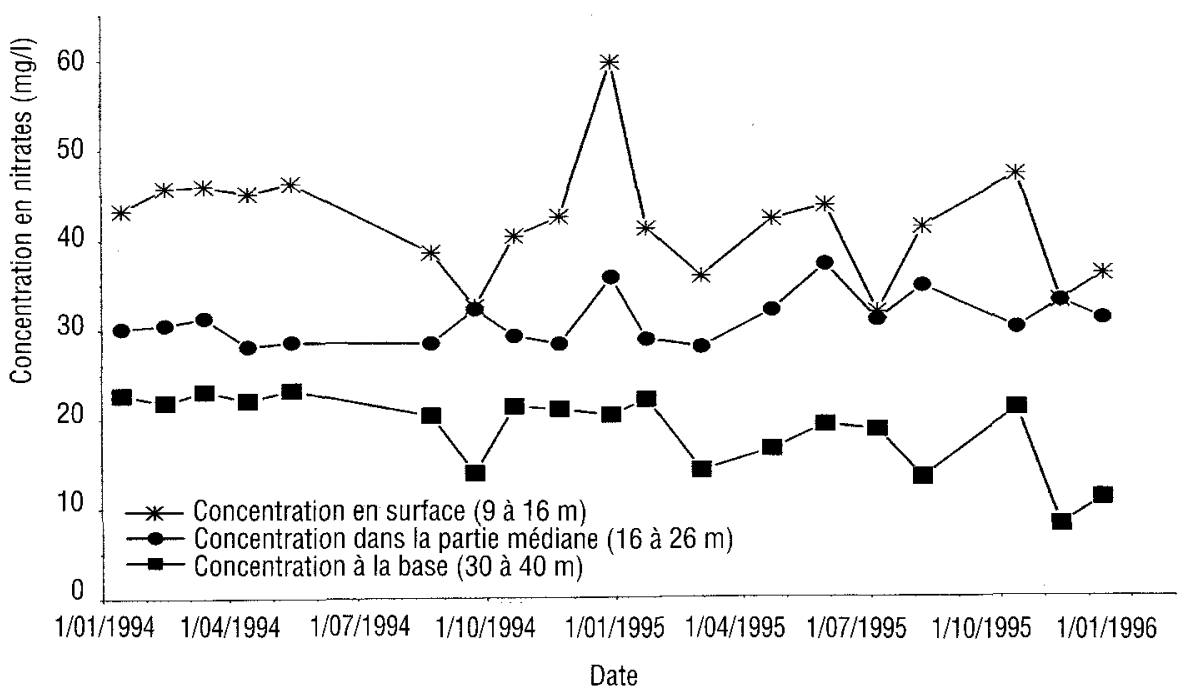

Figure 4 Évolution et répartition verticale des teneurs en nitrates en fonction de la profondeur (site de Bovenistier).

Evolution and vertical partitioning of nitrate levels in the Hesbaye aquifer (Bovenistier site). 


\section{4 - MODÉLISATION MATHÉMATIQUE DU TRANSFERT DES NITRATES EN MILIEU SATURÉ}

Devant l'impossibilité, pour des raisons d'ordre numérique (liées notamment aux nombres de Courant et de Peclet numériques), une modélisation des phénomènes d'écoulement et de transport à l'échelle régionale n'a pu être réalisée. Pour simuler, à long terme, l'évolution des teneurs en nitrates dans la nappe de Hesbaye, une région dont les caractéristiques géomorphologiques, géologiques et hydrogéologiques sont représentatives du plateau de Hesbaye a été sélectionnée. Située au nord (figure 1), elle s'étend selon un rectangle de $2,0 \mathrm{~km} \times 4,5 \mathrm{~km}$ allongé selon une direction nord-sud (figure 5). Sa superficie est couverte en majorité par des cultures de rotation, des zones de prairies peu étendues localisées autour des villages et de quelques villages. De plus, un bassin d'infiltration, recevant les eaux d'un collecteur d'égouts, est situé au sud de la zone. D'un point de vue géomorphologique, la région présente une vallée sèche importante correspondant à un axe d'écoulement préférentiel. L'épaisseur des terrains non saturés varie de $20 \mathrm{~m}$ au sud à $5 \mathrm{~m}$ au nord.

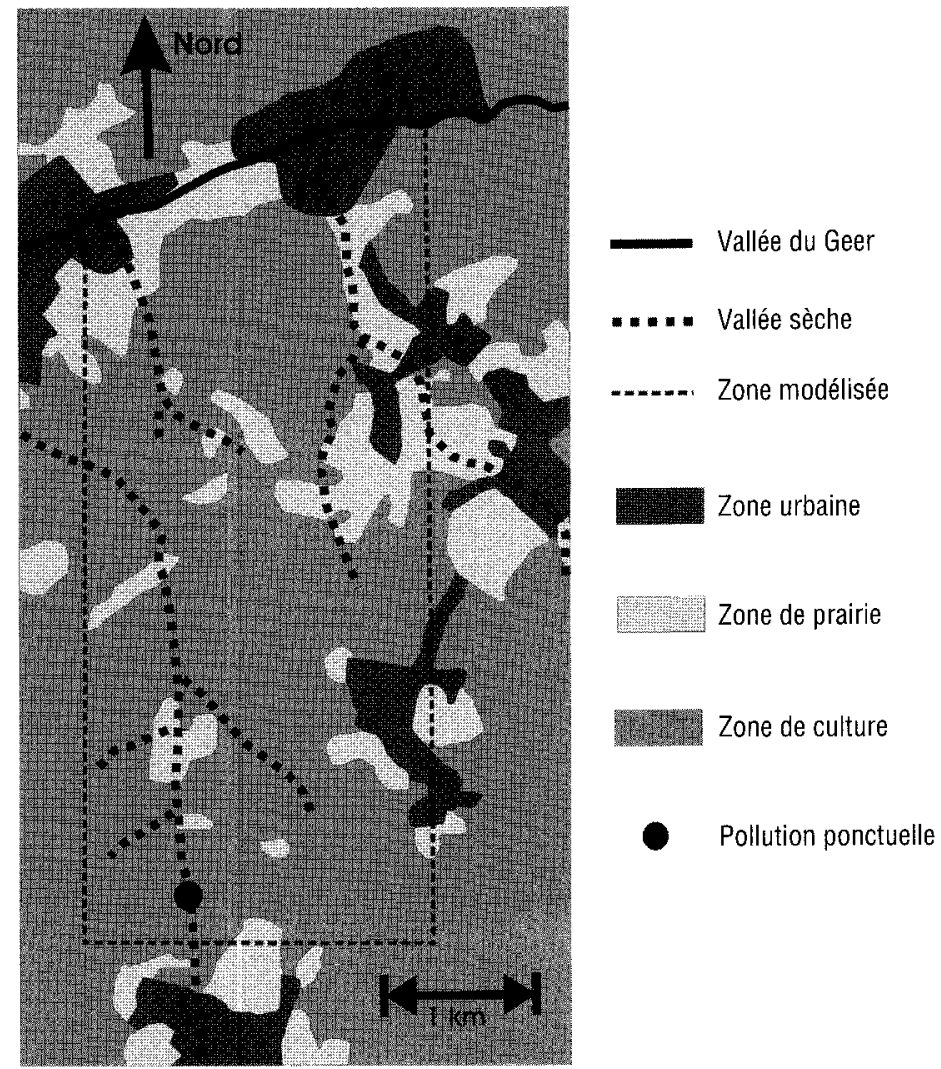

Figure 5 Carte d'occupation du sol.

Land use map. 
Le logiciel SUFT 3D (Saturated and Unsaturated Flow and Transport Model in 3D), développé par la Section Hydrogéologie du Département GEOMAC de I'Université de Liège a été utilisé. Ce logiciel résout, par la méthode des éléments finis, l'équation générale de l'écoulement et du transport de polluant dans un milieu saturé ou non (CARABIN et al., 1998). Il tient compte des phénomènes de convection, de dispersion, de diffusion, de dégradation et de l'effet d'eau immobile (BIVER et al., 1995).

Les éléments utilisés sont de type hexaédrique trilinéaire à 8 nœuds. La discrétisation et les conditions limites du modèle ont été réalisées à l'aide du logiciel GMS.

\subsection{Modèle conceptuel}

Des forages ( 3 puits et 10 piézomètres) ont permis de préciser la géométrie de l'aquifère. La zone modélisée a été discrétisée en 6 couches de 3350 éléments chacune. Les éléments ont une dimension de $50 \mathrm{~m} \times 50 \mathrm{~m}$. Verticalement, l'aquifère est subdivisé (de haut en bas) en :

- couche 1 : craies altérées au sud du modèle ou limon et conglomérat à silex dans la partie Nord (vallée du Geer), épaisseur de $3 \mathrm{~m}$ (région Sud) à $10 \mathrm{~m}$ (région Nord) ;

- couche 2 : craies altérées, épaisseur de $3 \mathrm{~m}$ à $8 \mathrm{~m}$;

- couche 3 : hardground, épaisseur de $2 \mathrm{~m}$;

- couches 4 à 6 : craies compactes ou fissurées en fonction de la présence ou non d'une vallée sèche ou d'un linéament ; chaque couche est épaisse de 10 à $15 \mathrm{~m}$.

Les terrains non saturés n'ont pas été discrétisés.

\subsection{Détermination des paramètres hydrodynamiques et de transport}

Les pompages d'essais réalisés dans la région ont montré que les conductivités hydrauliques sont relativement élevées et homogènes. Neuf matériaux ont été définis; leur conductivité hydraulique varie de $0,810^{-4} \mathrm{~m} . \mathrm{s}^{-1}$ (hardground) à $8,010^{-4} \mathrm{~m}_{.} \mathrm{s}^{-1}$, valeurs proches de celles mesurées expérimentalement au droit des différents puits présents dans la zone d'étude. Les plus fortes valeurs ont été attribuées aux craies situées au-dessus du hardground et aux craies situées dans l'axe d'une vallée sèche ou d'un linéament. Les paramètres d'écoulement ont été également définis à partir des valeurs obtenues lors de la calibration d'un modèle régional de la nappe de Hesbaye (HALLET et MONJOIE, 1996). Les valeurs des paramètres d'écoulement et de transport des différents matériaux (tableau 1) ont été obtenues à partir de 38 traçages répartis sur onze sites dont deux situés dans la zone modélisée (HALLET, 1997).

Des valeurs de dispersivités longitudinale et transversale élevées (respectivement $20 \mathrm{~m}$ à $40 \mathrm{~m}$ et $2,5 \mathrm{~m}$ à $4,0 \mathrm{~m}$ ) ont été attribuées aux différents matériaux. Ces valeurs tiennent compte de l'échelle régionale à laquelle le modèle simule les transports de polluants et des fortes valeurs des perméabilités des craies démontrant que ces dernières sont assez altérées, facteur favorisant une plus forte dispersion. Les craies altérées, situées au-dessus du hardground, sont les plus perméables. 
Les porosités efficaces varient de $2 \%$ à $5 \%$; les valeurs les plus élevées sont attribuées aux craies situées au-dessus du hardground.

Le coefficient d'échange entre l'eau mobile et l'eau immobile a été fixé à $1.10^{-7} \mathrm{~s}^{-1}$ et la porosité d'eau immobile à $10 \%$ pour la craie inférieure et à $25 \%$ pour la craie supérieure altérée.

La dégradation par dénitrification a été supposée nulle dans la région, les teneurs en oxygène atteignant plus de $4 \mathrm{mg} . \mathrm{I}^{-1}$, valeurs supérieures aux conditions de dénitrification liées à la présence de conditions anoxiques (MARIOTTI, 1994).

Tableau 1 Valeur des paramètres d'écoulement et de transport appliqués au modèle régional

Table 1 Flow and transport parameters used for the regional modelling

\begin{tabular}{ccccccccc}
\hline Matériaux & Nature & $\begin{array}{c}\mathbf{K} \\
\left(\mathbf{m} . \mathbf{s}^{-1}\right)\end{array}$ & $\mathbf{n}_{\mathrm{e}}$ & $\mathbf{S}$ & $\begin{array}{c}\alpha_{1} \\
(\mathbf{m})\end{array}$ & $\begin{array}{c}\alpha_{1} \\
(\mathbf{m})\end{array}$ & $\begin{array}{c}\alpha_{\mathbf{d}}{ }^{*} \\
\left(\mathbf{s}^{-1}\right)\end{array}$ & $\theta_{\text {imm }}$ \\
\hline 1 & craie supérieure & $8.010^{-4}$ & 0.05 & $1.10^{-3}$ & 40 & 4.0 & $1.10^{-7}$ & 0.25 \\
2 & craie supérieure & $5.010^{-4}$ & 0.05 & $1.10^{-3}$ & 40 & 4.0 & $1.10^{-7}$ & 0.25 \\
3 & hardground & $8.010^{-5}$ & 0.01 & $1.10^{-3}$ & 25 & 2.5 & $1.10^{-7}$ & 0.25 \\
4 & craie inférieure fissurée & $4.410^{-4}$ & 0.02 & $1.10^{-3}$ & 30 & 3.0 & $1.10^{-7}$ & 0.25 \\
5 & craie inférieure & $2.010^{-4}$ & 0.02 & $1.10^{-3}$ & 30 & 3.0 & $1.10^{-7}$ & 0.10 \\
6 & craie inférieure altérée & $4.010^{-4}$ & 0.02 & $1.10^{-3}$ & 30 & 3.0 & $1.10^{-7}$ & 0.10 \\
7 & craie inférieure peu altérée & $3.010^{-4}$ & 0.02 & $1.10^{-3}$ & 20 & 2.0 & $1.10^{-7}$ & 0.10 \\
8 & craie inférieure & $1.010^{-4}$ & 0.02 & $1.10^{-3}$ & 30 & 3.0 & $1.10^{-7}$ & 0.10 \\
9 & limon & $3.010^{-6}$ & 0.02 & $1.10^{-3}$ & 30 & 3.0 & $1.10^{-7}$ & 0.10 \\
\hline
\end{tabular}

$\mathrm{K}$ : conductivité hydraulique ; ne : porosité efficace ; $\mathrm{S}$ : coefficient d'emmagasinement ;

$\alpha_{1}$ : dispersivité longitudinale ; $\alpha_{t}$ : dispersivité transversale; $\alpha_{d}$ : coefficient d'échange entre l'eau mobile et l'eau immobile; $\theta_{\text {imm }}$ : porosité d'eau immobile.

\subsection{Détermination des conditions aux limites}

Pour l'écoulement, les conditions aux frontières sont les suivantes:

- au sud : conditions de potentiel imposé variant selon un pas de temps mensuel en accord avec les fluctuations piézomètriques saisonnières mesurées dans un piézomètre situé à $200 \mathrm{~m}$ de la frontière sud du modèle ;

- au nord: la frontière du modèle se situe le long du Geer (figure 1 et figure 2) et une condition de potentiel imposé y a été définie. La cote du lit du Geer varie linéairement de $99 \mathrm{~m}$ à l'amont à $94,8 \mathrm{~m}$ à l'aval ;

- à l'ouest et à l'est, les frontières sont supposées imperméables ; l'étude des cartes piézométriques montre que les isopièzes sont perpendiculaires (écoulement cylindrique en direction de la rivière drainante) aux frontières du modèle ;

- à la base : la base de l'aquifère est imperméable (Smectite de Herve) ;

- au sommet : les flux d'infiltration d'eau, estimés à partir d'un bilan hydrologique, ont été représentés par des conditions de Cauchy. 
Pour le transport, une condition de Cauchy a été appliquée à la surface du modèle, afin de simuler les flux de nitrates entrant au sommet de la nappe (voir ci-dessous). Ailleurs, une condition de Neumann nulle a été appliquée : le polluant entre ou quitte le domaine par convection à la concentration du milieu.

\subsection{Détermination des sollicitations}

Les flux en provenance de la surface ont été déterminés selon un pas de temps journalier à l'aide du modèle EPIC (Erosion Productivity Impact Calculator, US Department of Agriculture, 1990) utilisé par l'Unité d'Hydraulique Agricole des Facultés de Gembloux (DAUTREBANDE et al., 1996). Ces flux (figure 6 et figure 7) sont fonction :

- des précipitations et de l'infiltration efficace ;

- de l'occupation du territoire (cultures de rotation, prairies, zones urbaines) ;

- de l'épaisseur des terrains non saturés (les flux ont été calculés pour des épaisseurs de $1,5 \mathrm{~m}, 5 \mathrm{~m}, 10 \mathrm{~m}, 15 \mathrm{~m}$ et $20 \mathrm{~m}$ ).

Treize classes d'infiltration ont ainsi été définies :

- quatre valeurs d'intrant sous cultures de rotation : les flux varient de $110^{-7}$ à $710^{-7} \mathrm{mg} \cdot \mathrm{m}^{-2} \cdot \mathrm{s}^{-1}$ en fonction de la variation de l'épaisseur des terrains non saturés (5 à $20 \mathrm{~m})$ :

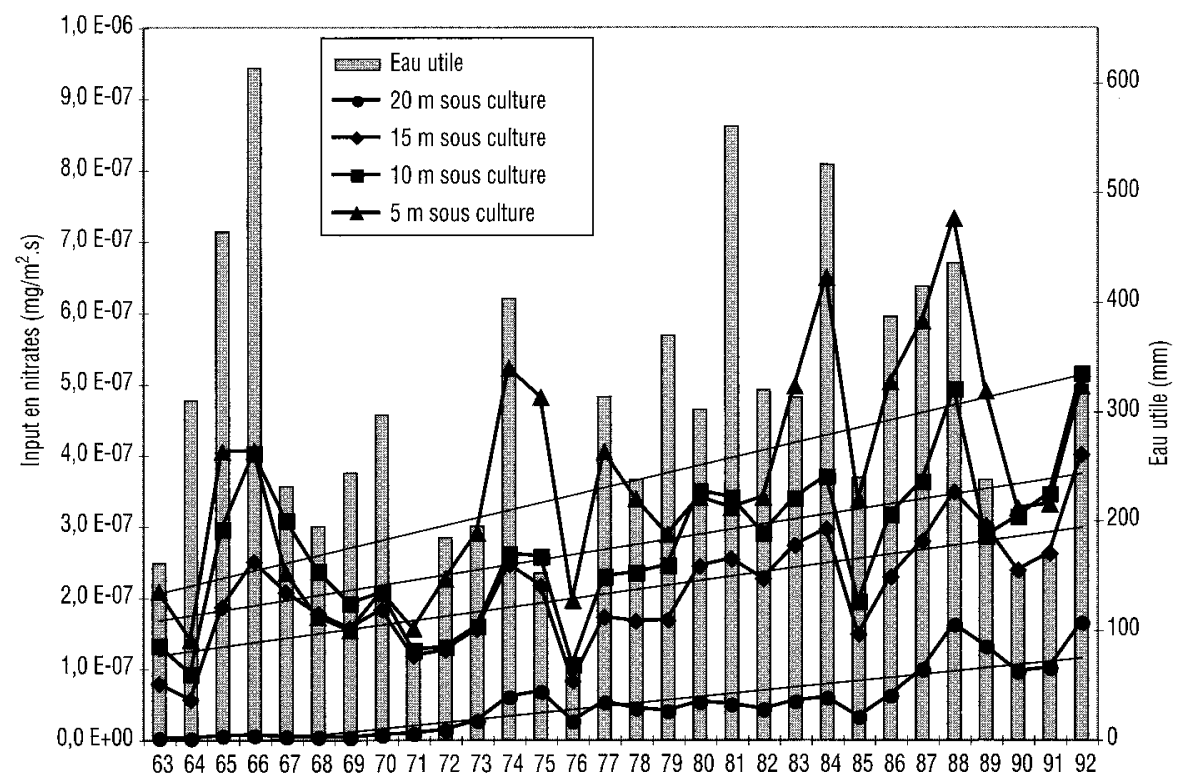

Année

Figure 6 Évolution des flux de nitrates sous cultures de rotation (valeurs moyennes annuelles).

Nitrate input flux under crop areas (mean annual values). 


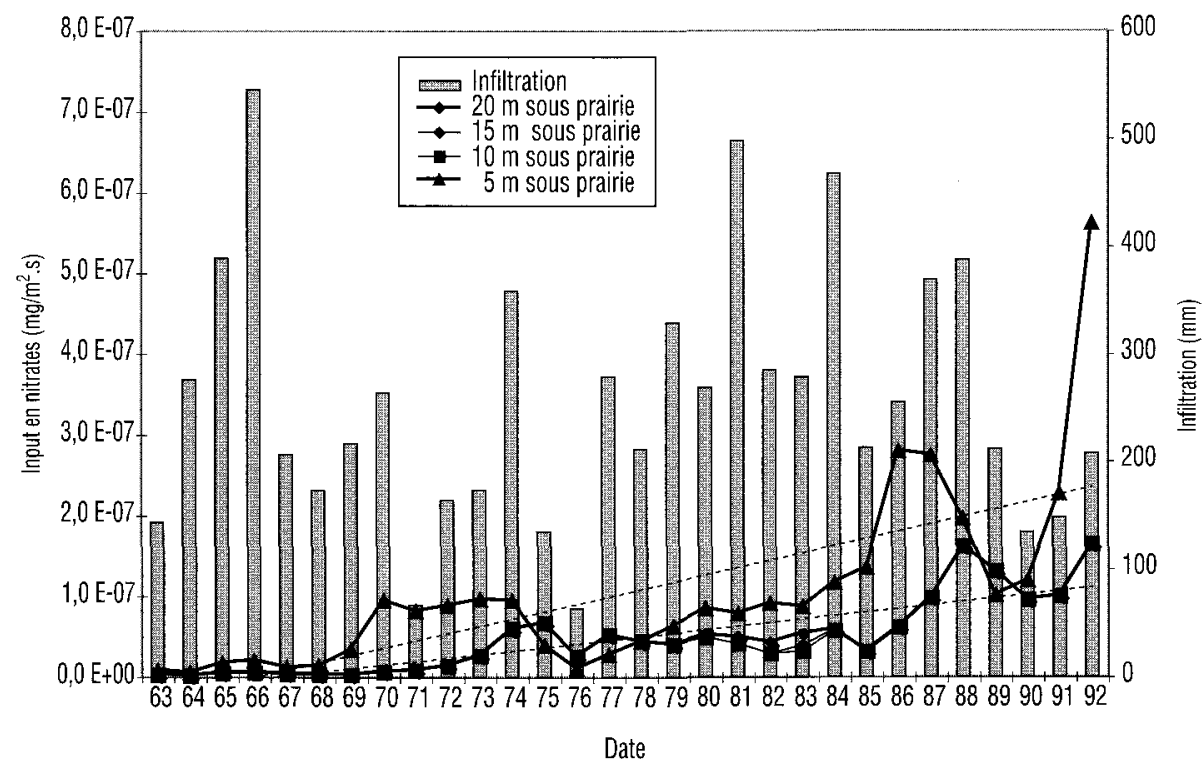

Figure 7 Évolution des flux de nitrates sous prairies (valeurs moyennes annuelles).

Nitrate input flux under grassland (mean annual values).

- quatre valeurs d'intrants sous prairies ; les flux restent néanmoins inférieurs à $5,410^{-7} \mathrm{mg} \cdot \mathrm{m}^{-2} \cdot \mathrm{s}^{-1}$.

- quatre valeurs d'intrant en nitrates sous les villages de Oreye, Otrange, Thys et Fize-le-Marsal (fonction de la densité de population et de la présence ou non d'un réseau d'égouttage) ; les valeurs de flux varient de 0,9 à $8,510^{-8} \mathrm{mg} \cdot \mathrm{m}^{-2}$. $\mathrm{s}^{-1}$; ils restent constants dans le temps, la population n'ayant pas évolué de manière significative durant les trente dernières années ;

- une valeur d'intrant liée à la contamination de la nappe au droit de l'exutoire des égouts de Momalle (bassin d'infiltration); teneurs moyennes de $2,4410^{-5} \mathrm{mg} \cdot \mathrm{m}^{-2} \cdot \mathrm{s}^{-1}$.

Le captage de Fize-le-Marsal, appartenant à la Société Wallonne de Distribution d'Eau, exploite la nappe à raison de $42 \mathrm{~m}^{3} \cdot \mathrm{h}^{-1}$.

L'évolution des teneurs en nitrates dans la nappe a été simulée sur la période de 1963 à 1992.

\subsection{Conditions initiales}

Préalablement à toute simulation, il est nécessaire de définir les concentrations initiales en nitrates de la nappe. En utilisant les intrants calculés en 1963, une simulation sur 70 années a été nécessaire pour obtenir une stabilisation des concentrations en nitrates vers $14 \mathrm{mg} \cdot \mathrm{l}^{-1}$ (figure 8), valeur proche des concentrations moyennes mesurées à l'échelle régionale à cette époque (voir figure 3). 


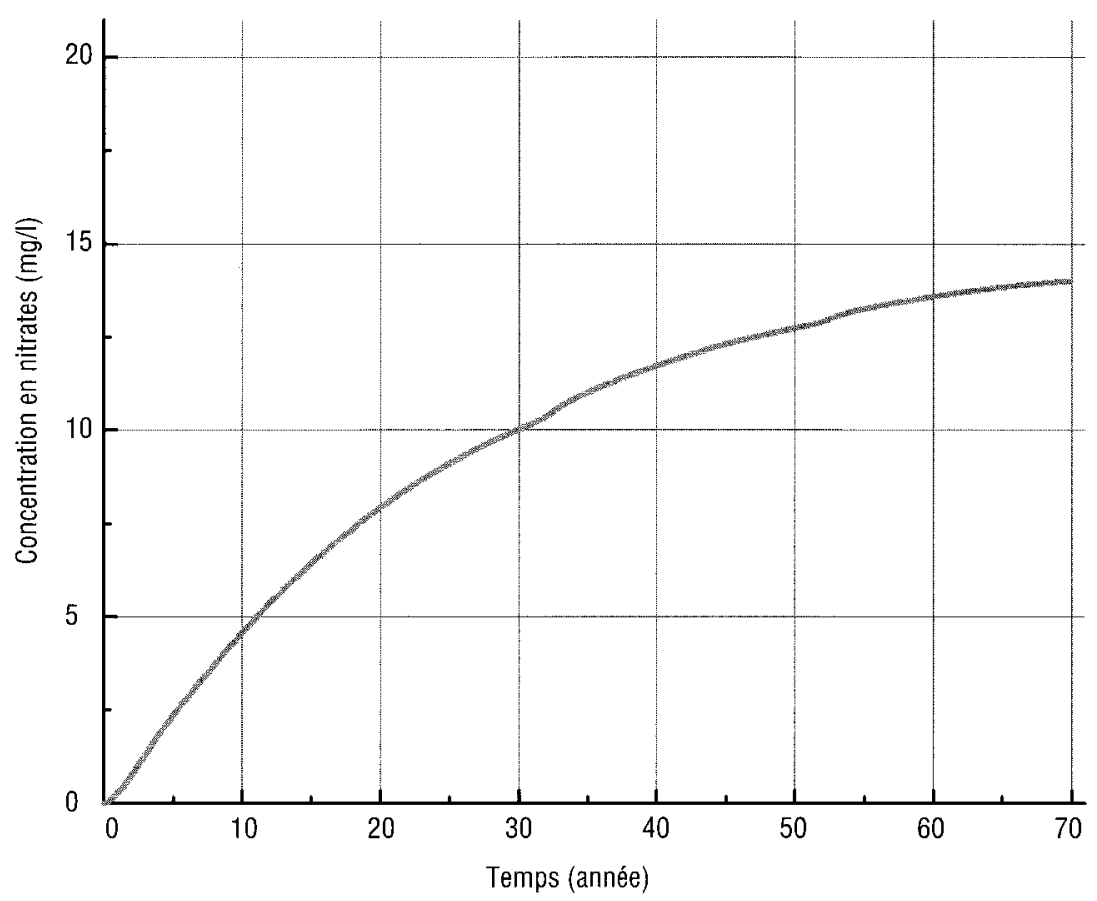

Figure 8 Simulations préliminaires : évolution des teneurs simulées en nitrates (site de Fize-le-Marsal).

Initial simulations : evolution of nitrate content (Fize-le-Marsal site).

\section{5 - RÉSULTATS DES SIMULATIONS}

Deux séries de simulations ont été réalisées (HALLET, 1999 b) : la première en simulant, de 1963 à 1992, les apports d'origine agricole uniquement afin de bien mettre en évidence les effets liés aux pollutions diffuses d'origine agricole, la seconde en simulant, durant la même période, les apports d'origine agricole et les apports liés à un bassin d'infiltration situé dans la partie amont du modèle (pollution ponctuelle).

Pour ces simulations, les sollicitations et les conditions aux frontières ont été définies mensuellement et le pas de temps de calcul était de une heure.

\subsection{Résultats des simulations relatives aux apports d'origine agricole (pollution diffuse)}

En 1963, des variations spatiales de concentrations sont observées (figure 9). Elles dépendent de la variabilité des apports de surface liés au 
contexte environnemental (occupation du sol, épaisseur des terrains non saturés). L'axe de la vallée sèche (coupe A-A') se marque par des teneurs en nitrates plus élevées, atteignant jusqu'à $16 \mathrm{mg} . \mathrm{l}^{-1}$. Au droit de cette vallée, les terrains non saturés sont moins épais qu'au droit des crêtes topographiques adjacentes; les flux de nitrates y sont donc plus importants $\left(0,910^{-7} \mathrm{mg} \cdot \mathrm{m}^{-2} \cdot \mathrm{s}^{-1}\right.$ au lieu de $0,0310^{-7}$ à $\left.0,0610^{-7} \mathrm{mg} \cdot \mathrm{m}^{-2} \cdot \mathrm{s}^{-1}\right)$. Les coupes transversales montrent qu'à partir de l'axe de cette vallée sèche, les nitrates migrent latéralement par dispersion et diffusion créant un gradient latéral de concentrations. En dehors de l'axe de la vallée sèche, les concentrations sont

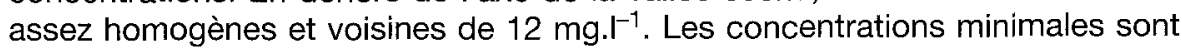
observées dans la partie nord-est du modèle, région de Thys (coupe D-D', partie Est), suite à la présence du village et de nombreuses prairies (faibles flux de nitrates en provenance de la surface soit $8.10^{-9} \mathrm{mg} \cdot \mathrm{m}^{-2} \cdot \mathrm{s}^{-1}$ pour le village de Thys et $2.10^{-8} \mathrm{mg} \cdot \mathrm{m}^{-2} . \mathrm{s}^{-1}$ sous les prairies). L'effet du captage de Fize-le-Marsal se marque par une légère déflexion vers l'est de la zone contaminée à partir de la vallée sèche (point $F$ ).

Fin 1992, suite à l'augmentation des flux de nitrates d'origine agricole, les concentrations dans la nappe augmentent et varient entre de 10 à $30 \mathrm{mg}^{-1} \mathrm{l}^{-1}$ (figure 10). Les concentrations maximales sont toujours observées au droit de la vallée sèche, située dans la partie ouest du modèle et les concentrations minimales au droit des crêtes topographiques, situées dans la partie est. La vue en plan montre clairement les effets liés aux apports de surface :

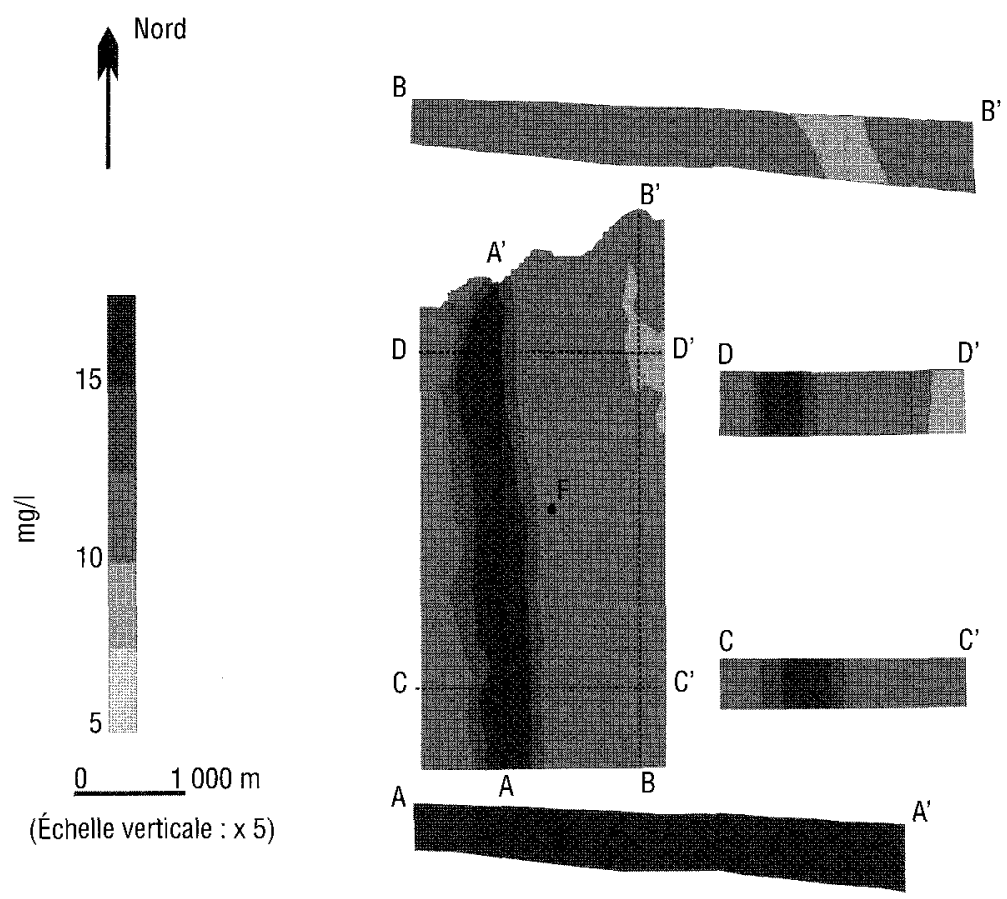

Figure 9 Répartition de teneurs simulées en nitrates en 1963.

Nitrate content map: the situation in 1963. 


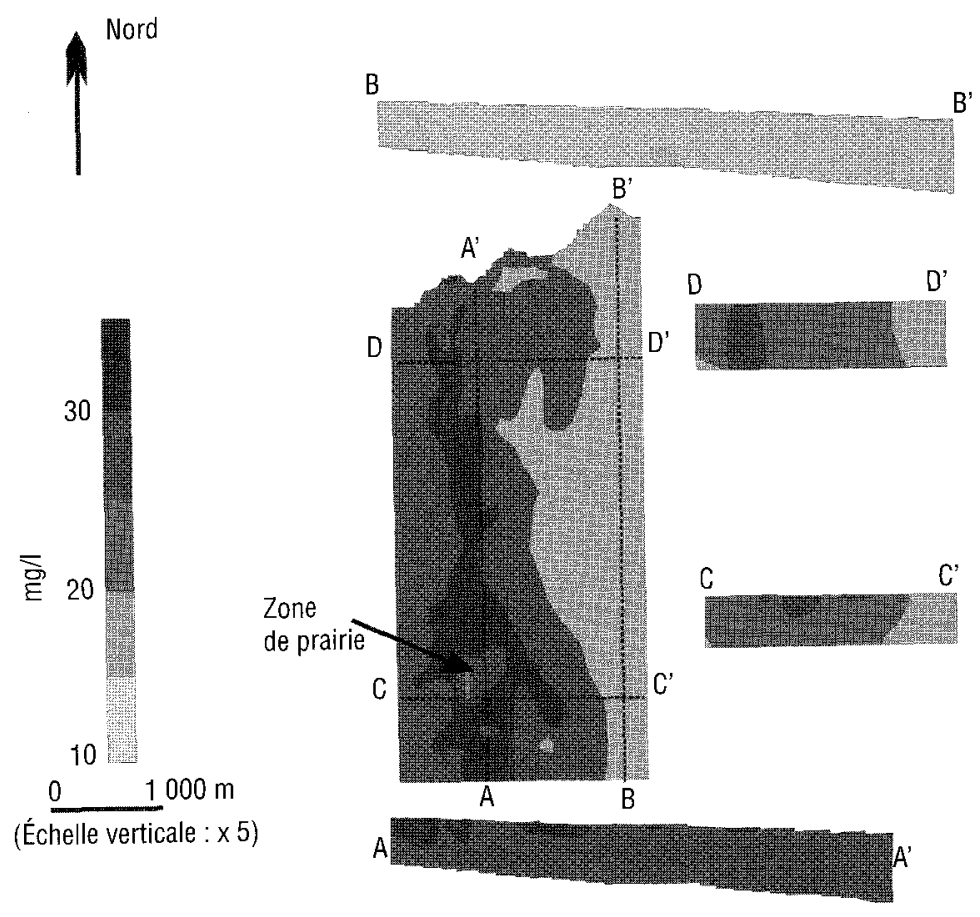

Figure 10 Répartition des teneurs simulées en nitrates en 1992 (pollution diffuse).

Nitrate content map: 1992 simulation (regional contamination).

- dans l'axe de la vallée sèche, les zones de prairies correspondent aux zones de plus faibles concentrations;

- les plus faibles concentrations, observées dans la partie est du modèle, sont liées aux zones urbaines ou sont dues, comme dans la partie sudest, à la plus grande profondeur de la nappe (15 à $20 \mathrm{~m}$ ).

Les différentes coupes longitudinales et transversales montrent l'existence d'un faible gradient vertical de concentrations (voir coupe A-A'), avec une diminution des concentrations avec la profondeur principalement au droit des zones fortement contaminées (écoulement subhorizontal en sommet de nappe limitant l'extension verticale de la pollution).

\subsection{Résultats des simulations relatives aux apports d'origine agricole (pollution diffuse) et ponctuelle (bassin d'infiltration)}

La simulation de l'évolution des concentrations des teneurs en nitrates d'origines agricole et ponctuelle montre que pour l'année 1992 (fin de la période simulée) les teneurs en nitrates dans la nappe varient entre 12 et plus de $35 \mathrm{mg}^{-1}$ (figure 11). 
Nord
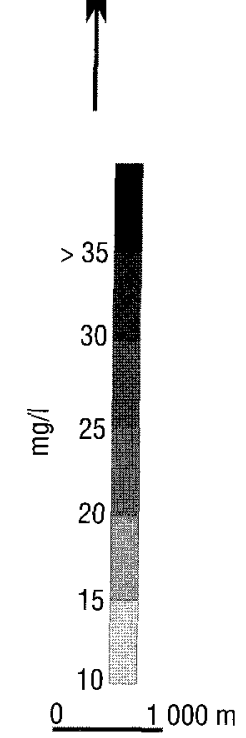

(Échelle verticale : $\times 5$ )

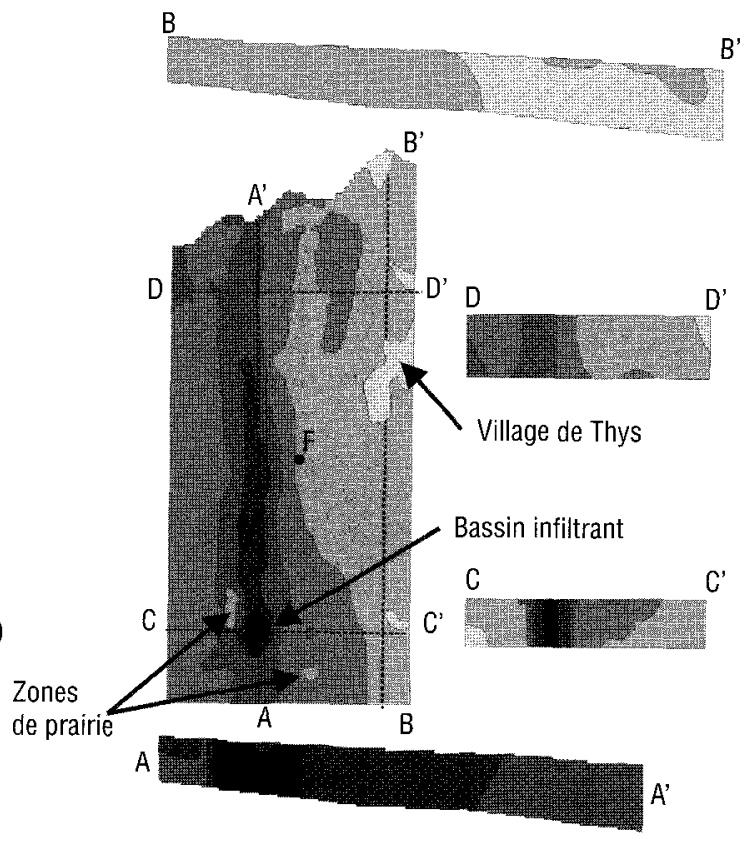

Figure 11 Répartition des teneurs simulées en nitrates en 1992 (pollutions ponctuelles et diffuses).

Nitrate content map: 1992 situation (point-source and regional contamination).

La vue en plan montre clairement :

- la contamination liée au bassin d'infiltration bien localisée dans l'axe de la vallée sèche au droit de laquelle des concentrations supérieures à $30 \mathrm{mg}^{-1} \mathrm{I}^{-1}$ (en gris foncé sur la figure 11) s'étend jusqu'à plus de 2,5 kilomètres en aval ; sous le bassin d'infiltration, les concentrations atteignent $50 \mathrm{mg}^{-1} \mathrm{I}^{-1}$;

- les concentrations plus faibles sont à nouveau observées au droit des crêtes topographiques, situées dans la partie est du modèle ;

- l'effet du pompage de la SWDE à Fize-le-Marsal (point F) se marque par une légère déflexion vers l'est du nuage de polluant ;

- dans l'axe de la vallée sèche principale, les zones de plus faibles concentrations correspondent à l'emplacement des prairies ;

- de faibles concentrations (15 $\left.\mathrm{mg} . \mathrm{I}^{-1}\right)$ sont observées au droit des zones urbaines égouttées suite aux plus faibles flux de nitrates vers la nappe (village de Thys). 


\section{3 Évolution des concentrations en nitrates de 1963 à 1992 et simulations prévisionnelles}

L'évolution des teneurs en nitrates de 1963 à 1992 montre qu'à l'aval de la pollution ponctuelle, les teneurs en nitrates varient fortement en fonction de la piézométrie (figure 12). II s'agit d'un phénomène de dilution, puisqu'en période de hautes eaux, le gradient régional augmente et les flux d'eau souterraine amènent au droit du site une plus grande quantité d'eau de plus faible concentration, diluant ainsi la pollution qui, elle, reste constante car indépendante de l'infiltration efficace (flux constant d'origine anthropique). Cette situation se marque particulièrement bien dans les années 1968, 1984 et 1989 (périodes de hautes eaux) durant lesquelles une baisse significative des concentrations est observée et, inversement, en 1965, 1974, 1978 et 1992 (périodes de basses eaux), années à plus fortes concentrations en nitrates. À noter que l'amplitude des fluctuations est plus importante à la base qu'au sommet de la nappe.

Les concentrations sont plus élevées à la surface de la nappe qu'en profondeur. Une différence de $10 \mathrm{mg} . \mathrm{l}^{-1}$ est observée entre les concentrations calculées au sommet du hardground et celles calculées à la base de la nappe soit un gradient de l'ordre de $0,5 \mathrm{mg} . \mathrm{l}^{-1}$ par mètre de profondeur.

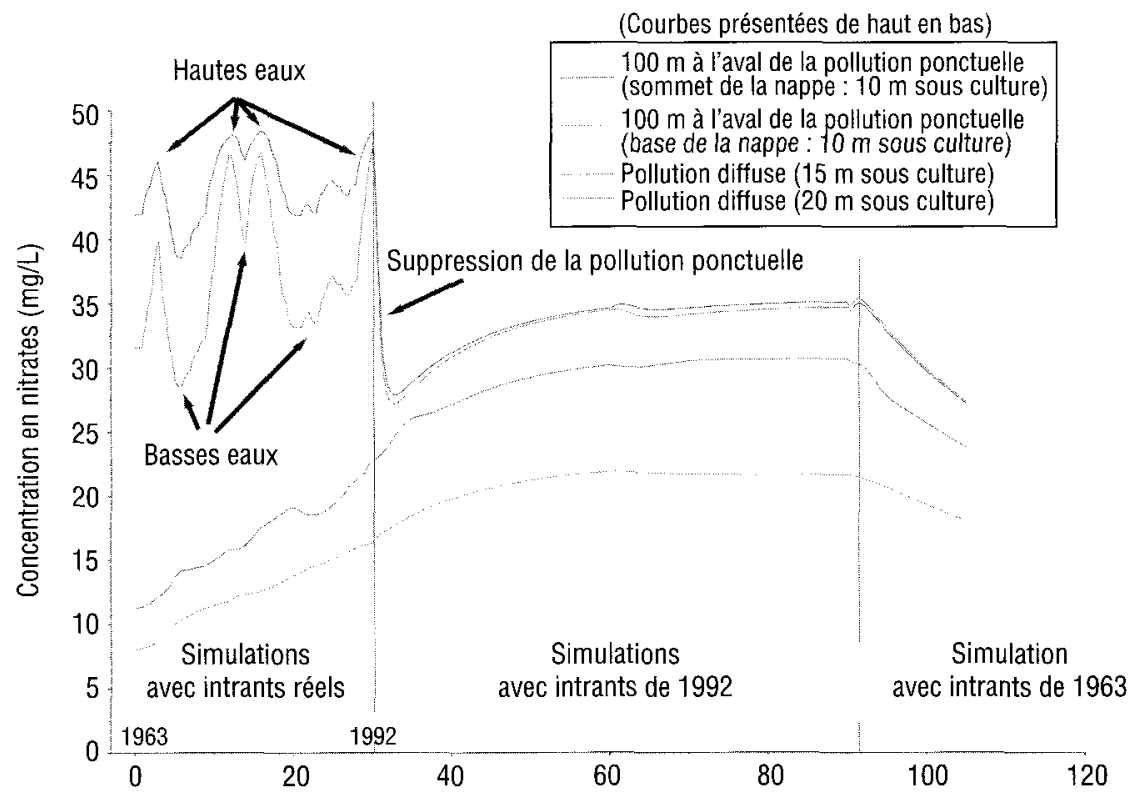

Figure 12 Simulation de l'évolution des concentrations en nitrates de 1963 à 1992 et simulations prévisionnelles.

Simulated aquifer nitrate content from 1963 to 1992 and future forecasts. 
Hors de la zone contaminée par la pollution ponctuelle, l'évolution des concentrations est nettement moins influencée par les phénomènes saisonniers. Le modèle montre que suite à l'augmentation des intrants (d'un facteur 4 en moyenne sur la zone modélisée soit de $1,210^{-7}$ en 1963 à $5,1410^{-7} \mathrm{mg} \cdot \mathrm{m}^{-2} \cdot \mathrm{s}^{-1}$ en 1992) les concentrations augmentent assez régulièrement (de 0,5 mg. $\mathrm{I}^{-1}$ par an, valeur proche de celle observée sur le terrain).

En ce qui concerne l'évolution à long terme des concentrations, deux situations ont été simulées : dans un premier temps, les infiltrations au droit du bassin ont été supprimées à partir de 1992 et les flux de nitrates d'origine agricole ont été maintenus constants et égaux à ceux de 1992 ; ensuite les flux de nitrates ont été ramenés à ceux de 1963. L'évolution des teneurs en nitrates en certains nœuds du modèle (figure 12) montre que les teneurs en nitrates diminuent très rapidement en aval du bassin d'infiltration des égouts de Momalle et la pollution, de même que l'entièreté du panache, sont résorbés en deux ans. Suite aux apports d'origine agricole (pour rappel, estimés constants à partir de 1992), les teneurs en nitrates dans la nappe continuent à augmenter à un rythme de $0,2 \mathrm{mg}^{-1} \mathrm{I}^{-1}$ par an durant une trentaine d'années. Après 60 ans de simulations, les concentrations se stabilisent entre 33 et $20 \mathrm{mg}^{-1} \mathrm{I}^{-1}$ selon la localisation du site (vallée sèche ou crête topographique).

Après stabilisation, les simulations ont été poursuivies durant 15 ans, en appliquant au sommet de la nappe les flux tels que définis en 1963. Les concentrations décroissent lentement $\left(0,7 \mathrm{mg} . \mathrm{I}^{-1}\right.$ par an) et devraient tendre, logiquement, après une cinquantaine d'années, vers des valeurs proches de celles définies comme conditions initiales du modèle. Ce temps de réaction important entre les concentrations des flux diffus entrant et la concentration à laquelle la nappe s'équilibre est due au caractère de la craie qui présente une double porosité : la porosité efficace étant, dans la région étudiée de l'ordre de 0,05 tandis que la porosité dite d'eau immobile peut atteindre jusqu'à 0,25 . La très lente décroissance des courbes de restitution, observées lors des traçages expérimentaux, confirme ce phénomène.

\section{6 - CONCLUSIONS}

Suite à l'augmentation constante des teneurs en nitrates dans la nappe aquifère de Hesbaye cette dernière a été décrétée "zone vulnérable " par le Gouvernement wallon. Afin de mettre en place des mesures de protection efficaces, une étude interdisciplinaire a été réalisée. L'étude hydrogéologique a permis de préciser la dynamique des nitrates dans la nappe. La synthèse de nombreuses données disponibles depuis 1956 montre que les concentrations en nitrates dans la nappe sont fortement tributaires du contexte hydrogéologique (nappe libre ou semi-captive), de l'occupation du sol (zone urbaine, de prairie ou de culture de rotation) et des fluctuations piézométriques saisonnières. Pour les pollutions ponctuelles, les concentrations sont, suite au phénomène de dilution, minimales en périodes de hautes eaux et, inversement, maximales en périodes de basses eaux. Pour les pollutions diffuses d'origine 
agricole, la nappe présente des concentrations minimales en périodes de basses eaux suite à un décrochement du front de nitrates qui migre plus lente-

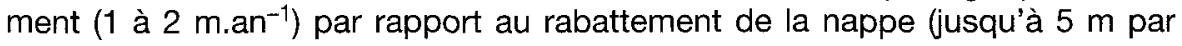
an).

La détermination des paramètres d'écoulement et de transport à partir de plus de 35 traçages répartis sur onze sites a permis de construire un modèle d'écoulement et de transport régional.

Les résultats du modèle montrent que la nappe réagit de manière distincte selon que la pollution est ponctuelle ou diffuse. Pour une pollution ponctuelle, la nappe réagira dans un délai de 1 à 2 ans à toute modification des intrants. Par contre, pour les pollutions d'origine diffuse, une à deux décennies sont nécessaires pour que les concentrations de la nappe s'équilibrent avec ces nouveaux intrants. Ces résultats, en terme de temps de réaction, sont primordiaux pour définir les mesures de prévention à mettre en cuvre. La suppression de pollutions ponctuelles conduira à une amélioration rapide mais locale de la qualité des eaux souterraines; la suppression des pollutions diffuses, d'origine agricole dans le cas de la Hesbaye, nécessitera des programmes de gestion à long terme de l'occupation du territoire et des rejets d'effluents d'élevages. Cette lente décroissance des concentrations est liée à la double porosité de la craie et aux effets d'eau immobile qui en découlent, elle ne peut être généralisée à tout type d'aquifère. Notons que les résultats ne concernent que des simulations en milieu saturé ; au temps de réaction de la nappe estimé par le modèle doit s'ajouter le temps de transit du polluant au travers de la zone non saturée. Des études, et notamment des traçages en milieu non saturé, ont par ailleurs été réalisées et sont en cours d'interprétation.

\section{REMERCIEMENTS}

Cette étude a été réalisée lors du «Programme Action Hesbaye - Programme Life ", financé par la Communauté Européenne, la Région Wallonne, la Compagnie Liégeoise de Distribution d'Eau et la Société Wallonne de Distribution d'Eau. 


\section{RÉFÉRENCES BIBLIOGRAPHIQUES}

BEAR J. et BACHMAT Y. (1990) - Introduction to modelling of transport phenomena in porous media. Kluwer Academic Publishers, Norwell, $553 \mathrm{p}$.

BIVER P., HALLET V. et DASSARGUES A. (1995) - Contribution to the simulation of nitrates transport in a double-porosity aquifer, I.A.H., Congress XXVI : Solutions'95, Edmonton-Canada.

CARABIN G., DASSARGUES A. et BROUYĖRE S. (1998). 3D flow and transport groundwater modelling including river interactions, in Computational Methods in Water Resources XII, Vol 1 : Computational Methods in Contamination and Remediation of water Resources, Burganos V.N., Karatzas G.P., Payatakes A.C., Brebbias C.A., Gray W.G. and Pinder G.F. Eds, pp. 569576, Computational Mechanics Publication.

DASSARGUES A. et MONJOIE A. (1993) Chalk as an aquifer in Belgium. In hydrogeology of the chalk of North-West Europe, OXFORD UNIVERSITY PRESS, chapter 8: pp. 153-169.

DAUTREBANDE S., DEWEZ A., HALLET V., GUIOT J., ROUXHET F., MONJOIE A. 1996. Rapport scientifique du Programme Action Hesbaye. Ministère de la Région wallonne, DGRNE. Service des Eaux Souterraines, $167 \mathrm{p}$.

DAUTREBANDE S., DEWEZ A., CASSE C., HENNEBERT P. (1999) - Nitrate leaching at regional scale : an explicit example of the hydrotope concept application. Modelling of transport processes in soils at various scales in time and space. EurAgEng Soll and Water Interest Group's International Workshop, Leuven, Belgium.
HALLET V. (1997) - Caractérisation des paramètres hydrodynamiques et hydrodispersifs de la craie du Crétacé de Hesbaye (Belgique). Bull. Soc. Géol. du Nord. Fasc 3, T. 5, pp 305-310.

HALLET V. (1999a) - Évolution et répartition des nitrates dans la nappe aquifère de Hesbaye (Belgique). Tribune de l'eau, $n^{\circ} 4$ \& 5. Vol 52. $N^{\circ}$ 600-601, pp. 51-58.

HALLET V. (1999b) - Étude de la contamination de la nappe de Hesbaye par les nitrates : hydrogéologie, hydrochimie et modélisation mathématique des processus d'écoulement et de transport en milieu saturé. Thèse de doctorat en Sciences ; Université de Liège, Belgique, $361 \mathrm{p}$.

HALLET V., SCHROEDER C., MONJOIE A. (1997) - Détermination des hétérogénéités du milieu crayeux par prospection géophysique. Bull. Soc. Géol. Du Nord. Fasc 3, T. 5, pp. 331-336.

HALLET V. et MONJOIE A. (1996) - Rapport scientifique du Programme Action Hesbaye. Volume LGIH. Ministère de la Région wallonne, DGRNE. Service des Eaux Souterraines.

MANGIN A. (1984) - Pour une meilleure connaissance des systèmes hydrologiques à partir des analyses corrélatoires et spectrales. Journal of Hydrology, $\mathrm{n}^{\circ} 67$, pp. 25-43.

MARIOTTI A., 1994. Dénitrification in situ dans les eaux souterraines, processus naturels ou provoqués: une revue. Hydrogéologie, $\mathrm{n}^{\circ} 3$, pp. 43-68.

US Department of Agriculture. (1990) Agriculture Research Service. Technical Bulletin, $\mathrm{n}^{\circ} 1768$. 